\title{
Implantation of Osmotic Pumps and Induction of Stress to Establish a Symptomatic, Pharmacological Mouse Model for DYT/PARK-ATP1A3 Dystonia
}

\author{
Lisa Rauschenberger ${ }^{1}$, Susanne Knorr ${ }^{1}$, Jens Volkmann ${ }^{1}$, Chi Wang Ip ${ }^{1}$
}

${ }^{1}$ Department of Neurology, University Hospital of Würzburg

\section{Corresponding Author}

Chi Wang Ip

Ip_C@ukw.de

\section{Date Published}

September 12, 2020

\section{Citation}

Rauschenberger, L., Knorr, S., Volkmann, J., Ip, C.W. Implantation of Osmotic Pumps and Induction of Stress to Establish a Symptomatic, Pharmacological Mouse Model for DYT/ PARK-ATP1A3 Dystonia. J. Vis. Exp. (163), e61635, doi:10.3791/61635 (2020).

DOI

$10.3791 / 61635$
URL

jove.com/video/61635

\section{Abstract}

Genetically modified mouse models face limitations, especially when studying movement disorders, where most of the available transgenic rodent models do not present a motor phenotype resembling the clinical aspects of the human disease. Pharmacological mouse models allow for a more direct study of the pathomechanisms and their effect on the behavioral phenotype. Osmotic pumps connected to brain cannulas open up the possibility of creating pharmacological mouse models via local and chronic drug delivery. For the hereditary movement disorder of rapidonset dystonia-parkinsonism, the loss-of-function mutation in the $\alpha 3$-subunit of the $\mathrm{Na}^{+} / \mathrm{K}^{+}$-ATPase can be simulated by a highly specific blockade via the glycoside ouabain. In order to locally block the a3-subunit in the basal ganglia and the cerebellum, which are the two brain structures believed to be heavily involved in the pathogenesis of rapidonset dystonia-parkinsonism, a bilateral cannula is stereotaxically implanted into the striatum and an additional single cannula is introduced into the cerebellum. The cannulas are connected via vinyl tubing to two osmotic pumps, which are subcutaneously implanted on the back of the animals and allow for the chronic and precise delivery of ouabain. The pharmacological mouse model for rapid-onset dystonia-parkinsonism carries the additional advantage of recapitulating the clinical and pathological features of asymptomatic and symptomatic mutation carriers. Just like mutation carriers of rapid-onset dystonia parkinsonism, the ouabain-perfused mice develop dystonia-like movements only after additional exposure to stress. We demonstrate a mild stress paradigm and introduce two modified scoring systems for the assessment of a motor phenotype. 


\section{Introduction}

The advantages of a continuous drug delivery directly into the brain are numerous. Repetitive and frequent injections, which represent an unnecessary stress factor for animals, can be avoided and a more constant intracerebral concentration of the drug can be achieved. This is especially valid when systemically administered drugs fail to easily penetrate the blood brain barrier. Moreover, chronic drug delivery via osmotic pumps allows for the localized delivery of substrates that would otherwise have system-wide side effects. The drugs can be delivered in a targeted manner to the desired brain structures, the resulting effect can thus be directly traced. This can be utilized for an array of applications, such as the study of therapeutic effects as well as the study of pathomechanisms. This last application was used in the project herein in order to create a pharmacological mouse model for dystonia.

The analysis and understanding of dystonic syndromes, which represent the third most common movement disorder, have been heavily limited by the fact that genetic animal models largely fail to reproduce the disease phenotype found in diseased human as well as the pathophysiology. This issue is not limited to dystonic syndromes, but in fact concerns many transgenic rodent models in the field of movement disorders ${ }^{1,2}$. The reason for the lack of a phenotype in transgenic rodent models might be based on highly effective compensatory mechanisms ${ }^{3}$. In the case of dystonia, the disease is characterized by involuntary muscle contractions causing twisting movements and abnormal postures ${ }^{4}$. The study of secondary causes (i.e., brain injury) of dystonic symptoms, has helped to identify the structures involved in the manifestation of these motor abnormalities, such as the basal ganglia ${ }^{5}$. Brain imaging studies of hereditary forms of dystonia have shown functional abnormalities in almost all brain regions responsible for motor control and sensorimotor integration 6,7 . However, rodent models are still needed to deepen the understanding of the neural dysfunctions on a molecular and large scale network level as well as for the development of therapeutic options. This is where pharmacological mouse models offer the possibility to replicate the clinical and pathological features of a disease in a more precise manner.

Rapid-onset dystonia-parkinsonism (DYT/PARK-ATP1A3; RDP; DYT12) is one of the hereditary forms of dystonia. It is caused by loss-of-function mutations in the ATP $1 \alpha 3$ gene, which encodes for the a3-subunit of the $\mathrm{Na}^{+} / \mathrm{K}^{+}$ATPase $^{8}$. Furthermore, it is recognized that gene mutation carriers can be free of symptoms for years before acutely developing persistent generalized dystonia and Parkinsonism after exposure to a stressful event. Indeed, the penetrance of DYT/PARK-ATP1A3 is incomplete and stressful events acting as a trigger range from physical overexertion and extreme temperatures to overconsumption of alcohol and infections $^{9}, 10$. In order to study DYT/PARK-ATP1A3 and to find potential therapeutic interventions, it has been tried numerous times to imitate the stress-dependent disease development in rodent models. However, aside from the one existing genetic DYT/PARK-ATP1A3 mouse model, where transient abnormal and convulsion-like movements were induced by hypothermia, all published genetic mouse models for DYT/PARK-ATP1A3 have failed to produce dystonic symptoms ${ }^{1,11,12}$. Calderon et al. previously demonstrated that blocking the a3-subunit bilaterally in the basal ganglia and the cerebellum via the cardiac glycoside ouabain in wild type mice results in mild gait disturbance ${ }^{13}$. The additional exposure to electrical foot shocks in a warm environment led to a dystonic and bradykinetic phenotype, thus demonstrating that chronic and targeted perfusion of 
ouabain followed by stress successfully imitates the DYT/ PARK-ATP1A3 phenotype.

However, exposing animals to electrical foot shocks in a warm environment of $38-40{ }^{\circ} \mathrm{C}$ over a two hour-period induces pain and anxiety in animals, which represent confounding factors, especially for the assessment of changes in the catecholamine system related to the development of dystonia. Thus, we herein describe a different kind of stress paradigm with high translational value, which relates back to the fact that mild to moderate exercise have been described as triggers in DYT/PARK-ATP1A3 patients ${ }^{9}$. Moreover, repetitive exercise is a well-known trigger for focal dystonia $^{14}$. Mice were repeatedly subjected to challenging motor tasks comprised of three descends of a wooden pole ("pole test") and three runs on a Rotarod apparatus ("Rotarod performance test"). The placement of animals on the top of a $50 \mathrm{~cm}$ wooden pole was used to coerce the animals to descend, the Rotarod apparatus was employed to subject mice to forced activity by placing them on a rotating rod.

The characterization of the motor phenotype of a mouse model for dystonia is particular challenging due to the lack of predefined tests and scores. However, one variation of a motor disability assessment has been repeatedly used over the last years in order to evaluate the severity and the distribution of dystonia-like movements in rodents ${ }^{13}, 15,16$ We herein present a modified version of the dystonia rating scale, which proved to be effective in evaluating the dystonialike phenotype of animals when observed over a time period of four minutes. As a second method of assessing dystonialike movements, we present a newly-developed scoring system for the assessment of abnormal movements during a tail suspension test. It allows for the assessment of the frequency and duration of dystonia-like movements and postures of the front limbs, hindlimbs as well as trunk.

\section{Protocol}

All procedures were performed in accordance with applicable international, national, and/or institutional guidelines for care and use of animals. The local authorities at the Regierung von Unterfranken, Würzburg, Germany, approved all animal experiments.

\section{Priming of osmotic pumps}

NOTE: This step has to be performed at least $48 \mathrm{~h}$ prior to surgery. ALZET osmotic pumps need to be prefilled in order to ensure that the pumping rate reaches a steady state before implantation.

1. Prepare the desired solution for chronic perfusion beforehand. Ensure that the solvent and agent are compatible with the osmotic pumps and catheters. For the project herein, thaw a $10 x$ stock solution of ouabain (stored at $-20^{\circ} \mathrm{C}$ ) at room temperature and vortex for $10 \mathrm{~s}$. CAUTION: Ouabain is toxic: it should be handled only with gloves and opened only under a sterile hood to avoid inhalation.

2. Turn the sterile hood on; disinfect the hood as well as all the instruments and material needed before putting them under the hood.

3. Put on surgical gloves before handling the osmotic pumps. Separately prepare the ouabain-solution designated for the pumps of the striatum and the cerebellum.

NOTE: Consider that the concentration of the solution in the pump for the striatum has to be doubled compared to the concentration of the solution in the pump designated 
for the cerebellum. This is due to the fact that the pump for the striatum is connected to a double cannula, the flow rate, however, is the same as for the pump of the cerebellum, which is connected to a single cannula.

4. Calculate the amount of solution needed using the following formula:

mass delivery rate $\left(\mathrm{k}_{\mathrm{O}}\right)=$ volume delivery rate $(\mathrm{Q}) \mathrm{x}$ concentration of the agent in the vehicle $\left(C_{d}\right)$

For the present project, the osmotic pumps were primed and filled with either ouabain solution at a concentration of $11.2 \mathrm{ng} / \mathrm{h}$ or $0.9 \%$ saline for the control group.

5. Vortex both ouabain solutions for $10 \mathrm{~s}$ and sterile filter (i.e., $0.22 \mu \mathrm{m}$ syringe-end filter) them into separate, new microcentrifuge tubes, using a different filter for each solution.

6. Weigh the empty osmotic pumps together with the flow moderator (about $0.4 \mathrm{~g}$ ).

7. Fill a $1 \mathrm{~mL}$ syringe with a $27 \mathrm{G}$ filling cannula (preferably with a blunt tip) with sterile filtered ouabain-solution or vehicle solution.

8. Get all the air bubbles out of the syringe, hold the osmotic pump in an upright position, insert the cannula all the way into the pump and slowly fill the reservoir until excess solution appears on top (avoid rapid filling as this can lead to air bubbles in the pump). Next, insert the flow moderator into the pump (excess solution should appear on top).

9. Pull the flow moderator out (about $5 \mathrm{~mm}$ ), carefully take off the white flange with scissors and connect a piece of vinyl tubing to the flow moderator. Ensure that the catheter has a length of approximately $2 \mathrm{~cm}$ to allow proper mobility of the animal.
10. Weigh the filled osmotic pump once more to ensure that the difference in weight between filled and empty pump is concordant with the expected weight of the solution loaded.

NOTE: For most aqueous solutions this weight is equal to the volume in microliters. If the weight does not correspond to the expected volume, air might be trapped inside the pump, which needs to be emptied and refilled.

11. For the priming of the pumps, prepare two $2 \mathrm{~mL}$ microcentrifuge tubes, one tube for the striatum and one for the cerebellum.

12. Submerge the pumps into the microcentrifuge tubes filled halfway with $0.9 \%$ saline solution. Do not submerge the open end of the catheter. Put the microcentrifuge tubes into a thermocycler for $48 \mathrm{~h}$ at $37^{\circ} \mathrm{C}$ in order to ensure that the pump has reached a steady pumping rate before implantation and the catheters are prefilled.

\section{Cannula and osmotic pump implantation}

1. Place the mouse (male, $\mathrm{C} 57 \mathrm{BI} / 6 \mathrm{~N}, 11-12$ weeks of age) into a chamber designed for inhalation anesthesia; set the flow rate of isoflurane to $2-3 \%$ and the flow rate of oxygen to $2 \mathrm{~L} / \mathrm{min}$.

2. After the mouse is deeply anaesthetized according to approved protocols, shave the top of the animal's head, dorsal neck and proximal third of the back.

3. Place the animal into a stereotactic frame and continue the anesthesia with isoflurane via a mouse anesthesia mask designed for the stereotactic instrument (isoflurane flow rate $1.5-2 \%, 2 \mathrm{~L} / \mathrm{min}$ oxygen).

4. Using rubber tips or non-rupture ear bars, fix the head of the animal in the stereotactic frame, taking care that the head is leveled. 
5. In order to prevent hypothermia, place a heating pad under the animal, insert a rectal temperature probe and set the temperature to $37^{\circ} \mathrm{C}$. Protect the eyes of the animal from drying out by using a drop of ophthalmic ointment on each eye.

6. Apply an analgesic, such as Carprofen $(5 \mathrm{mg} / \mathrm{kg}$ bodyweight), subcutaneously before beginning surgery.

7. With a syringe subcutaneously inject up to $0.2 \mathrm{~mL}$ of bupivacaine $0.25 \%$ and wait $30 \mathrm{~s}$ for the local anesthesia to take effect.

8. Disinfect the shaved areas thoroughly with an antiseptic, such as octenidine dihydrochloride.

9. After thorough disinfection of the surgical area, use a scalpel to place an incision at the top of the head and use scissors to continue the incision down to the fore limbs. Expose the skull with the help of bulldog clamps and wipe the periosteum with a sterile cotton-tipped applicator.

10. Align either a pen or the tip of a cannula stained in black ink with bregma and use the appropriate coordinates to mark the three entry points for the brain cannulas on the skull (coordinates for the bilateral holes needed for perfusion of the basal ganglia: anterior/posterior: + $0.74 \mathrm{~mm}$, medial/lateral: $+/-1.50 \mathrm{~mm}$ (+ indicating the right side, - the left side relative to bregma); coordinates for the hole at the midline of the cerebellum: anterior/ posterior: $-6.90 \mathrm{~mm}$ ). Next, carefully drill the holes for the double cannula designated for the basal ganglia and the single cannula designated for the cerebellum (Figure 1A).

11. Drill a fourth hole for a small screw in-between the striatum and the cerebellum. This screw will eventually be embedded in dental cement and provide additional hold for the cannulas. Using fine forceps and a screw driver, carefully introduce the screw into the small hole until it is firmly fixed in the skull. Do not implant the screw too deeply as this damages the brain tissue.

NOTE: It is recommended to continue with the osmotic pump implantation before inserting the osmotic cannulas. This avoids causing any accidental damage to the cannulas when implanting the pumps.

12. In order to create a small pocket for the osmotic pump on each side of the animals back, use tissue forceps to separate the subcutaneous tissue layers. Advance the forceps towards one hind leg and open the forceps slightly in order to widen the subcutaneous pocket. Repeat the same procedure for the other side, first removing the forceps from the incision and then pushing them gently towards the second hind leg.

NOTE: The pocket should permit the pump to easily slide in, however, it should not have too much room to move under the skin.

13. With the help of tissue forceps take the first pump together with the connected piece of tubing and slide it into one subcutaneous pocket. Repeat the same procedure with the second pump.

14. Using a minipump holder, introduce a single osmotic cannula with a custom length of $3.0 \mathrm{~mm}$ into the hole drilled in the midline of the cerebellum. Detach the cannula head carefully and fix the cannula as well as the small screw with dental cement, taking care as not to cover the connecting piece for the tubing of the osmotic pump. Ensure that the dental cement surrounding the cannula has fully dried before continuing with surgery.

15. Before inserting a double cannula with a center-to-center distance of $3.0 \mathrm{~mm}$ and custom-made length of $4.0 \mathrm{~mm}$ into the bilaterally drilled holes above the basal ganglia, attach two short pieces of vinyl tubing $(0.5 \mathrm{~cm})$ to the 
two connecting pieces of the double cannula. Connect the vinyl tubings with a bifurcation adaptor and carefully prefill the entire tubing system including the adaptor and cannula with ouabain solution or vehicle (room temperature, sterile filtered beforehand). This can be done best with a $1 \mathrm{~mL}$ syringe and a $27 \mathrm{G}$ filling cannula introduced into the single rear end of the bifurcation adaptor (Figure 1B).

16. Using a minipump holder, carefully insert the double cannula into the bilateral holes. Use a clamp to detach the cannula head and fix the double cannula with dental cement.

17. Connect the catheters of the osmotic pumps to the bifurcation adaptor as well as the single cannula, respectively. Ensure that the catheters have a strong hold on the connecting pieces of the cannulas.

NOTE: Both pumps have an equal flow rate, so be careful to connect the osmotic pump with the double concentrated solution to the bifurcation adaptor to ensure that the same concentration reaches both the basal ganglia and the cerebellum.

18. Close the incision on the back of the animals with stitches as far as possible in direction of the skull, being careful not to overstretch the skin (Figure 1C).

19. Subcutaneously inject $0.5 \mathrm{~mL}$ of $0.9 \%$ saline, which should have body temperature, into a skin fold on each side of the back of the animals in order to avoid dehydration of the mice, carefully avoiding the pumps.

\section{Motor challenge}

1. Subject ouabain- or vehicle-perfused mice to challenging motor tasks as a form of mild stress exposure $4 \mathrm{~h}$ postsurgery and repetitively every $24 \mathrm{~h}$ afterwards in order to induce dystonia-like movements. This does not include a behavioral characterization; its purpose is to induce a higher stress level compared to normal cage keeping.

2. Place the mouse onto a $50 \mathrm{~cm}$ rough-surfaced, wooden pole with a diameter of $1 \mathrm{~cm}$, nose facing downwards. Ensure that the pole is placed into a large cage with enough bedding in case of falls. It is not necessary to measure the time of descent, but look out for involuntary hyperextensions of front limbs and hindlimbs presented by ouabain-perfused animals while descending the pole. Let mice descend the pole three times and allow $2 \mathrm{~min}$ of recovery between descends.

NOTE: Ouabain-perfused mice should present first symptoms like bradykinesia $4 \mathrm{~h}$ post surgery. However, $24 \mathrm{~h}$ after surgery mice should start to present involuntary hyperextensions of front limbs and hindlimbs as a sign of dystonia-like movements during descend.

3. For the second motor task, place mice on the rotating rod as done for the Rotarod performance test. The Rotarod apparatus is not used as a measure of the latency to fall, the aim is to subject mice to forced activity. Place the mice on the rotating rod three times and allow 2 min of recovery between tests.

NOTE: To increase stress exposure, use an accelerating Rotarod apparatus. For the project described herein, the rod accelerates from 5 to $50 \mathrm{rpm}$ over a set time period of $300 \mathrm{sec}$.

4. Let animals recover for $30 \mathrm{~min}$ in between the pole test and the Rotarod performance test and again another 30 min before scoring for dystonia-like movements as described under protocol step 4. In between the repetitive stress exposures, allow mice to recover for $24 \mathrm{~h}$-intervals. 


\section{Scoring systems for the assessment of dystonia-like movements}

NOTE: The experimenter should be blinded to the group assignment analyzed to prevent bias. The behavioral tests used to characterize the phenotype of the mice are two scoring systems: a dystonia rating scale scoring abnormal, dystonia-like movements and a behavioral score using the tail suspension test. Assess the dystonia-like movements after a recovery time of $30 \mathrm{~min}$ following the exposure to mild stress.

1. Dystonia rating scale

NOTE: Due to the lack of predefined behavioral tasks, the dystonia rating scale was established as an observerbased scoring system similar to the clinical rating scales of human dystonia. It is a modified version of the dystonia rating scale used by Calderon et al. ${ }^{13}$.

1. Record posture and gait of animals over a period of 4 min after animals have been placed in a plastic or wooden box.

2. Score for frequency and distribution of dystonia-like movements from 0 to 4 points: (0) normal motor behavior; (1) abnormal motor behavior, no dystonialike movements; (2) mild motor impairment with mild focal dystonia-like movements; (3) moderate motor impairment with severe focal dystonia-like movements; (4) severe impairment, with sustained, generalized dystonia-like movements (Figure 2). Consider the following movements or postures as dystonia-like: hyperextension of front limbs, wide stance or hyperextension of hindlimbs as well as kyphosis. Consider dystonia as focal in case a single body part is affected and as generalized in case the trunk and at least two other body parts are affected.
2. Tail suspension test

NOTE: The tail suspension test is often used to observe and score for hindlimb clasping. This is however a highly unspecific phenotype indicating a motor impairment. The following protocol proposes a scoring system specific for dystonia-like movements. Due to the generalization of dystonia in DYT/PARK-ATP1A3 patients, dystonia-like movements should be assessed in front limbs, trunk and hindlimbs. A newly-developed scoring system from 0-8 points was developed, a total score $<2$ indicated that no dystonia-like movements were present (Figure 3).

1. Pick the mouse up by the tail near its base and lift the animal up. Record a 2 min video of the tail suspension test and assign a score in a subsequent, thorough analysis of the recording.

2. Score the front limbs from 0 to 4 points, where repeated or sustained tonic retractions of one or both front limbs, as well as a hyperextension combined with crossing of the front limbs, were classified as dystonia-like: (0) no abnormal movements; (1) reduced movement of front limbs with hyperextension of paws seen $\geq 50 \%$ of the recorded time; (2) mild dystonia-like movements of front $\operatorname{limb}(\mathrm{s})<50 \%$ of the recorded time; (3) mild dystonia-like movements of front $\operatorname{limb}(s) \geq 50 \%$ of the recorded time or severe $<50 \%$ of the recorded time; (4) severe dystonia-like movements of front $\operatorname{limb}(\mathrm{s}) \geq 50 \%$ of the recorded time.

NOTE: Hindlimb clasping is an abnormal movement that should not be scored as dystonia-like.

3. Score the hindlimbs from 0 to 3 points, where retraction and clenching of rear limbs as well as sustained hyperextension were assessed as 
dystonia-like: (0) no abnormal movements; (1) reduced movement of hindlimbs with hyperextension of paws seen $\geq 50 \%$ of the recorded time;

(2) dystonia-like movements of one hindlimb; (3) dystonia-like movements of both hindlimbs.

4. In case of truncal distortion $>80 \%$ of the recorded time, an additional point is added to the score.

5. Place the animal back into its cage.

\section{Representative Results}

Figure 4 has been modified from Rauschenberger et al. ${ }^{17}$. For data analysis of both the dystonia rating scale (A) and the tail suspension test $(B)$, calculate the total score for each time point for each animal. The mean of each time point and each group should be plotted on an appropriate graph. The distribution of the values should be investigated and the appropriate statistical test should be applied to determine significance. With a sufficient number of animals, a motor phenotype can be detected both with the dystonia rating scale as well as with the assessment of abnormal movements in the tail suspension test. The dystonia-like phenotype is demonstrated by the significantly higher motor score in both assessments in the ouabain-perfused, stressed group compared to ouabain-perfused, non-stressed mice as well as the control mice.
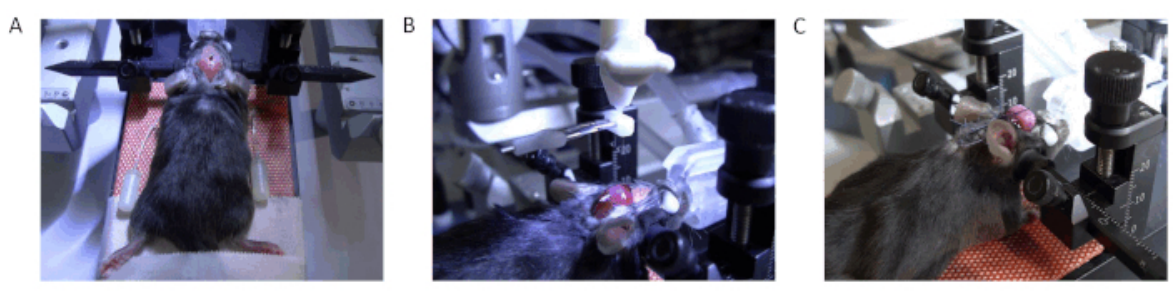

Figure 1: The main surgical steps for cannula and osmotic pump implantation. (A) For the indicated coordinates, holes need to be drilled bilaterally for the double cannula designated for the basal ganglia and for the single cannula placed at the midline of the cerebellum. The two fully-constructed osmotic pumps are shown on each side of the animal. (B) The picture shows an implanted, single cannula into the cerebellum, fixed with dental cement. The double cannula for the basal ganglia should be connected to the bifurcation adaptor and prefilled with ouabain before implantation. (C) Image of the finished procedure. Please click here to view a larger version of this figure. 


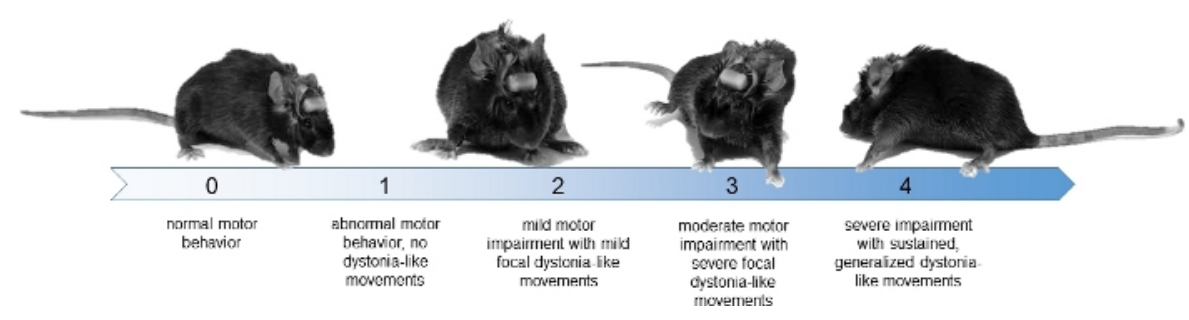

Figure 2: Assessment of dystonia-like movements with a dystonia rating scale. During a 4 min video, dystonia-like movements were scored based on body distribution and duration. Involuntary hyperextension of the front limbs, a wide stance or hyperextension of hindlimbs as well as kyphosis were rated as dystonia-like. Please click here to view a larger version of this figure.

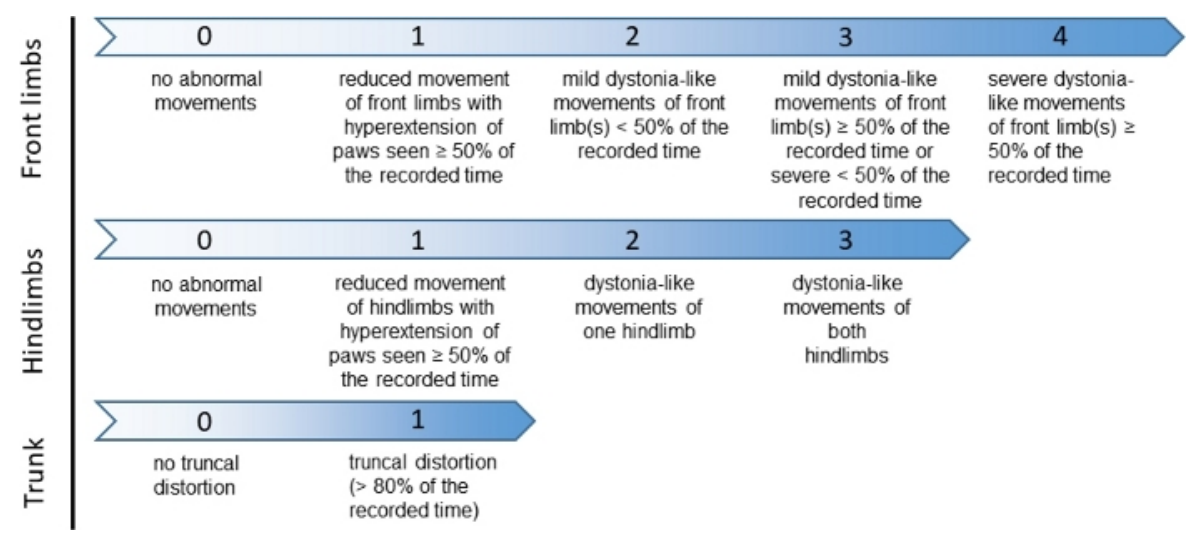

Figure 3: Assessment of dystonia-like movements during a tail suspension test. The newly-developed scoring system for abnormal movements during a 2 min tail suspension test evaluates dystonia-like movements in front limbs, hindlimbs and trunk from 0-8 points in total. For the front limbs, a hyperextension and crossing of the front limbs as well as a tonic flexion towards the trunk qualified as dystonia-like. For the hindlimbs the involuntary hyperextension as well as retraction with extension over the midline was scored as dystonia-like. A truncal distortion over $80 \%$ of the recorded time was scored with one point. Please click here to view a larger version of this figure. 


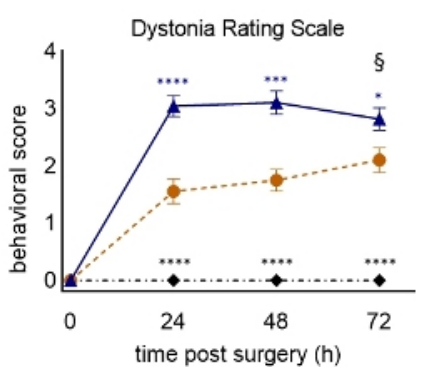

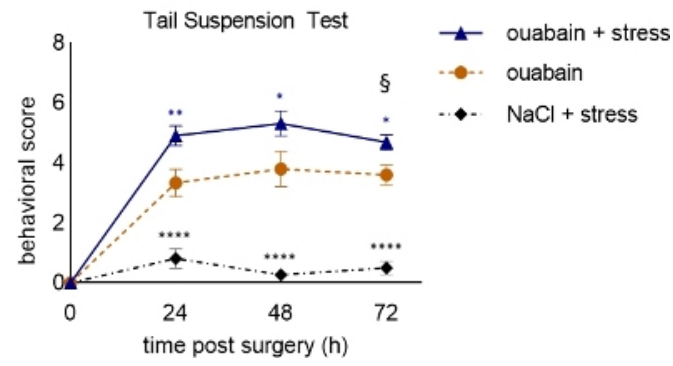

Figure 4: Representative graphs of the dystonia-rating scale and the tail suspension test. (A) The graph depicts the dystonia-rating scale for $\mathrm{NaCl}$-perfused, stressed mice (dotted black line), ouabain-perfused, non-stressed mice (dotted orange line) and ouabain-perfused, stressed mice (dark blue line). For each time point, the mean values \pm standard error of the mean (SEM) are shown. (B) The diagram shows the assessment of abnormal movements during a 2-min tail suspension test for $\mathrm{NaCl}$-perfused, stressed mice (dotted black line), ouabain-perfused, non-stressed mice (dotted orange line) and ouabain-perfused, stressed mice (dark blue line). Statistical analysis was done for the dystonia rating scale and the tail suspension test scoring using the two-tailed Mann-Whitney test. Bonferroni-Holm correction (§) of the p-values showed a significant difference for the observational period of $72 \mathrm{~h}$. Dark blue * denote significant differences between ouabainperfused, stressed mice and ouabain-perfused, non-stressed mice mice, black * denote significant differences between $\mathrm{NaCl}$-perfused, stressed mice and ouabain-perfused, stressed mice as well as between $\mathrm{NaCl}-$ perfused, stressed mice and ouabain-perfused, non-stressed mice. Please click here to view a larger version of this figure.

\section{Discussion}

This DYT/PARK-ATP1A3 pharmacological mouse model allows for the detailed analysis of intracerebral structural and neurochemical changes induced solely by inhibition of the sodium-potassium ion pump in the basal ganglia and cerebellum as well as alterations related to stress exposure. In case of mice, a maximum of two osmotic pumps can be subcutaneously implanted. We herein present a method detailing chronic drug delivery to multiple brain structures by implementing a double cannula connected to a bifurcation adaptor in addition to a single cannula. This methodology can be used for any application requiring multiple brain structures to be perfused simultaneously and chronically.
We present a mouse model of a rare movement disorder, where patients develop permanent symptoms after exposure to stress. This presumed gene-environmental interaction is still not well understood, but might represent one of the key pathomechanisms in DYT/PARK-ATP1A3 development. Different methods of exposing mice to stress have been published in the past and include electric foot shocks, restraining, cold or warm environment and exposure to various odors $11,12,13$. In an effort to expose mice to a mild stress factor with translational value, we herein describe the repetitive subjection of mice to challenging motor tasks. For the pole test, ouabain-perfused animals revealed involuntary hyperextension of front limbs and hindlimbs. These movements were very similar to the dystonia-like 
movements observed during the 4-min video recording of the animals as well as the tail suspension test. The application of mild stress in form of challenging motor tasks might prove useful in other mouse models showing motor symptoms or neurodegeneration, where gene-environmental interactions massively influence the degree of disease progression.

There is a general lack of predefined behavioural tasks as well as rating scales to classify abnormal movements and postures in mice. Most of the available motor tasks reveal unspecific abnormalities, such as hindlimb clasping, which is a well-known phenomenon in many mouse models of movement disorders with neurodegeneration ${ }^{18,19}$. For the proper characterization of a phenotype, it is however necessary to analyze whether the mouse model recapitulates the salient features of the disease. Herein, we present the modified version of a dystonia rating scale used previously for the assessment of motor disability in dystonia mouse models $^{15,16}$. We additionally developed an observer-based scoring system for the tail suspension test, which was established similarly to the clinical rating scales of human dystonia. Both rating scales show a significantly higher score in ouabain-perfused, stressed mice compared to ouabainperfused, non-stressed animals as well as vehicle-perfused animals. The drawbacks of any observer-based scoring system are the necessary training of raters to ensure consistent scoring and to reduce observer variability as well as the danger of a possible bias of the rater if not fully blinded to the group analysed. However, observer-based scoring systems still present an easily accessible method to characterize a phenotype and can be adapted to the mouse model analyzed, as done in the present project for the assessment of dystonia-like movements. To ensure consistent scoring among different raters, training videos should be made available. To reduce any potential bias, it is recommended that different raters score the same video clips and that the individual scores are averaged. Both scoring systems mentioned within this work record the presence of dystonia-like movements in animals. The rating scales can be adapted according to the specific requirements within a project, as done previously by Ip et al., where solely the hindlimbs were scored for dystonia-like movements in a mouse model for dystonia 1 (DYT-TOR1A) ${ }^{20}$. The rating scales can be complemented by other previously published scoring systems, assessing for example the degree of bradykinesia in rodents as done with the locomotion disability score by Calderon et al. ${ }^{13}$.

\section{Disclosures}

The authors have nothing to disclose.

\section{Acknowledgments}

This work was supported by the Federal Ministry of Education and Research (BMBF DysTract to C.W.I.) and by the Interdisciplinary Center for Clinical Research (IZKF) at the University of Würzburg (Z2-CSP3 to L.R.). The authors thank Louisa Frieß, Keali Röhm, Veronika Senger and Heike Menzel and for their technical assistance as well as Helga Brünner for the animal care.

\section{References}

1. DeAndrade, M. P., Yokoi, F., van Groen, T., Lingrel, J.B., Li, Y. Characterization of Atp1a3 mutant mice as a model of rapid-onset dystonia with parkinsonism. Behavioural Brain Research. 216 (2), 659-665 (2011).

2. Dang, M. T. et al. Generation and characterization of Dyt1 DeltaGAG knock-in mouse as a model for early-onset 
dystonia. Experimental Neurology. 196 (2), 452-463 (2005).

3. Kreiner, G. Compensatory mechanisms in genetic models of neurodegeneration: are the mice better than humans? Frontiers in Cellular Neuroscience. 956 (2015).

4. Albanese, A. et al. Phenomenology and classification of dystonia: a consensus update. Movement Disorders. 28 (7), 863-873 (2013).

5. Marsden, C. D., Obeso, J.A., Zarranz, J.J., Lang, A.E. The anatomical basis of symptomatic hemidystonia. Brain. 108 (Pt. 2), 463-483 (1985).

6. Carbon, M. et al. Increased sensorimotor network activity in DYT1 dystonia: a functional imaging study. Brain. 133 (Pt. 3), 690-700 (2010).

7. Carbon, M., Su, S., Dhawan, V., Raymond, D., Bressman, S., Eidelberg, D. Regional metabolism in primary torsion dystonia: effects of penetrance and genotype. Neurology. 62 (8), 1384-1390 (2004).

8. de Carvalho Aguiar, P. et al. Mutations in the $\mathrm{Na}+/ \mathrm{K}+-$ ATPase alpha3 gene ATP1A3 are associated with rapidonset dystonia parkinsonism. Neuron. 43 (2), 169-175 (2004).

9. Brashear, A. et al. The phenotypic spectrum of rapidonset dystonia-parkinsonism (RDP) and mutations in the ATP1A3 gene. Brain. 130 (3), 828-835 (2007).

10. Barbano, R. L. et al. New triggers and non-motor findings in a family with rapid-onset dystonia-parkinsonism. Parkinsonism \& Related Disorders. 18 (6), 737-741 (2012).

11. Isaksen, T. J. et al. Hypothermia-induced dystonia and abnormal cerebellar activity in a mouse model with a single disease-mutation in the sodium-potassium pump. PLOS Genetics. 13 (5), e1006763 (2017).

12. Sugimoto, H., Ikeda, K., Kawakami, K. Heterozygous mice deficient in Atp1a3 exhibit motor deficits by chronic restraint stress. Behavioural Brain Research. 272, 100-110 (2014).

13. Calderon, D. P., Fremont, R., Kraenzlin, F., Khodakhah, K. The neural substrates of rapid-onset DystoniaParkinsonism. Nature Neuroscience. 14 (3), 357-365 (2011).

14. Cutsforth-Gregory, J. K. et al. Repetitive exercise dystonia: A difficult to treat hazard of runner and nonrunner athletes. Parkinsonism \& Related Disorders. 27, 74-80 (2016).

15. Pizoli, C. E., Jinnah, H.A., Billingsley, M.L., Hess, E.J. Abnormal Cerebellar Signaling Induces Dystonia in Mice. The Journal of Neuroscience. 22 (17), 7825-7833 (2002).

16. Jinnah, H. A. et al. Calcium channel agonists and dystonia in the mouse. Movement Disorders. 15 (3), 542-551 (2000).

17. Rauschenberger, L., Knorr, S., Al-Zuraiqi, Y., Tovote, P., Volkmann, J., Ip, C.W. Striatal dopaminergic dysregulation and dystonia-like movements induced by sensorimotor stress in a pharmacological mouse model of rapid-onset dystonia-parkinsonism. Experimental Neurology. 323, 113109 (2020).

18. Fernagut, P. O., Diguet, E., Bioulac, B., Tison, F. MPTP potentiates 3-nitropropionic acid-induced striatal damage in mice: reference to striatonigral degeneration. Experimental Neurology. 185 (1), 47-62 (2004).

19. Guyenet, S. J. et al. A simple composite phenotype scoring system for evaluating mouse models of cerebellar 
ataxia. Journal of Visualized Experiments. 39, 1787 (2010).

20. Ip, C. W. et al. Tor1a+/- mice develop dystonia-like movements via a striatal dopaminergic dysregulation triggered by peripheral nerve injury. Acta Neuropathologica Communications. 4, 108 (2016). 